\title{
Fosinopril and Losartan Regulate Klotho Gene and Nicotinamide Adenine Dinucleotide Phosphate Oxidase Expression in Kidneys of Spontaneously Hypertensive Rats
}

\author{
Rong Tang $^{\mathrm{a}}$ Qiao-ling Zhou ${ }^{\mathrm{a}}$ Xiang Ao ${ }^{\mathrm{a}}$ Wei-sheng Peng ${ }^{\mathrm{a}}$ \\ Pouranan Veeraragoo ${ }^{a}$ Tian-feng Tang ${ }^{b}$ \\ ${ }^{a}$ Department of Nephrology, Xiangya Hospital, Xiangya Medical College, Central South University, Changsha, and \\ ${ }^{b}$ Department of Nephrology, Nanjing Drum Tower Hospital, The Affiliated Hospital of Nanjing University Medical \\ School, Nanjing, China
}

\section{Key Words}

Fosinopril $\cdot$ Losartan $\cdot$ Klotho $\cdot$ Nicotinamide adenine dinucleotide phosphate oxidase $\cdot$ Hypertensive renal damage

\begin{abstract}
Background/Aims: Klotho, a newly identified antiaging gene, predominantly detected in the kidney, has pleiotropic protective effects on kidney diseases. Several studies have confirmed the association between Klotho and oxidative stress. The present studies were performed to explore effects of fosinopril (Fos) and losartan (Los) on Klotho and nicotinamide adenine dinucleotide phosphate (NADPH) oxidase expression in kidneys of spontaneously hypertensive rats (SHR). Methods: Twenty-four male 22-week-old SHR were randomly divided into three groups: model group, Fos group and Los group. Wistar-Kyoto rats were taken as control. After 8 weeks, urinary $\mathrm{N}$-acetyl- $\beta$-D-glucosaminidase (NAGase), 24 h urinary protein (Upro), serum creatinine (Scr), blood urea nitrogen (BUN) and renal pathological changes were detected. Renal mRNA and protein expression of Klotho and three subunits of NADPH oxidase protein expression were evaluated. Results: As compared to the model
\end{abstract}

group, NAGase, Upro, Scr and BUN were decreased; the typical renal pathological damage was relieved in the Fos or Los group. Fos or Los inhibited the reduction of Klotho expression, and reduced the elevation of NADPH oxidase expression. Conclusion: Abnormal expression of Klotho and NADPH oxidase plays important roles in progression of hypertensive renal damage. Fos and Los can increase Klotho expression, and inhibit NADPH oxidase expression, which may be one of the mechanisms of their protective effects in hypertensive renal damage.

Copyright $\odot 2011$ S. Karger AG, Basel

\section{Introduction}

Klotho gene, with antiaging features and restricted organ distribution, is mainly expressed in the kidney tubules [1-3]. Several studies have found that the suppression of Klotho might contribute to the progression of renal diseases including acute renal failure, chronic renal failure, hypertensive renal damage, diabetic nephropathy, glomerulonephritis and renal cell carcinoma [4-9]. These findings indicate that Klotho gene is a protective gene in renal diseases. Antioxidative effect is one of the most pop-

\section{KARGER}

Fax +4161306 1234 E-Mail karger@karger.ch www.karger.com

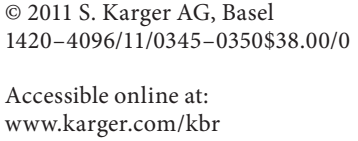

Prof. Qiao-ling Zhou, MD, PhD

Department of Nephrology, Xiangya Hospital

Xiangya Medical College, Central South University

Changsha, Hunan 410008 (China)

Tel. +86 7318432 7431, E-Mail zhouqling@yahoo.com.cn 
ular directions of research among molecular mechanisms of Klotho gene. Recent data showed that there was a close relationship between Klotho gene and oxidative stress [10, 11]. Klotho could induce resistance to oxidative stress at the cellular and organismal level in vitro and in vivo.

Accumulating evidence supports that oxidative stress characterized by overproduction of reactive oxygen species (ROS) and reduction of the antioxidative enzyme system is involved in the pathogenesis and progression of hypertensive renal damage [12]. Nicotinamide adenine dinucleotide phosphate (NADPH) oxidase is the primary enzyme of ROS production. NAPDH oxidase plays a critical role in the pathogenesis of hypertensive renal damage [13]. Klotho gene delivery could decrease renal superoxide production, which might be attributed to the suppression of Nox-2 and NADPH oxidase activity in spontaneously hypertensive rats (SHR) [6].

The renin-angiotensin system (RAS) plays a leading role in the development of hypertensive renal damage through its hemodynamic and non-hemodynamic effects, finally leading to interstitial fibrosis and chronic renal failure $[14,15]$. RAS inhibitors like angiotensinconverting enzyme inhibitors (ACEI) and angiotensin receptor blockers (ARB) are renoprotective beyond blood pressure lowering. However, the precise mechanisms of the renoprotective effects of ACEI and ARB in hypertensive renal damage remain to be elucidated.

Because Klotho gene and NADPH oxidase are thought to be involved in hypertensive renal damage, we adopted the animal model of SHR in vivo and drug intervention with fosinopril (Fos) or losartan (Los) to explore the possible mechanisms of their renoprotective effects in addition to antihypertensive ability in the current study.

\section{Methods}

\section{Experimental Animals}

All procedures were carried out within the institutional guidelines for animal research. Male SHR and Wistar-Kyoto (WKY) rats (200-250 g b.w.) were purchased at 22 weeks (SLAC, Shanghai, China). 24 SHR were randomly divided into three groups: SHR group (placebo, positive control), SHR + Fos group (fosinopril, $10 \mathrm{mg} / \mathrm{kg} /$ day), SHR + Los group (losartan, $20 \mathrm{mg} / \mathrm{kg} /$ day), and $8 \mathrm{WKY}$ rats treated with placebo acted as negative control. All treatments were administered orally once daily from 22 to 30 weeks of age. Systolic blood pressure (using tail-cuff plethysmography), urinary $\mathrm{N}$-acetyl- $\beta$-D-glucosaminidase (NAGase) and $24 \mathrm{~h}$ urinary protein (Upro) were measured at 22 and 30 weeks of age, respectively. The rats were sacrificed at 30 weeks of age under anesthesia with chloral hydrate $(3-4 \mathrm{ml} / \mathrm{kg}$, i.p.), blood was collected by cardiac puncture for serum creatinine (Scr) and blood urea nitrogen (BUN) evaluation. Finally, kidneys were collected and subjected to the studies described below.

\section{Morphological Studies}

For morphological experiments, left kidneys were fixed with $10 \%$ buffered formalin. Paraffin-embedded sections were prepared and stained with hematoxylin and eosin (HE). Histological scoring was performed in a blinded manner.

\section{Immunohistochemical Analysis for Klotho}

Kidney sections were fixed in formalin, then embedded in paraffin and serially sectioned $(3-4 \mu \mathrm{m})$ on a microtome. Thereafter, kidney sections were deparaffinated, rehydrated, microwaved, and stained using a standard three-layer method according to the SP kit. When examining Klotho, the first antibody was rabbit anti-rat Klotho antibody (1:100; US Biology, USA); the secondary antibody was rabbit-horseradish peroxidase (HRP)-conjugated antibody (1:2,000; Santa Cruz Biotechnology (SCB) Inc., Santa Cruz, Calif., USA). The bound peroxidase was mixed with diaminobenzidine to produce a brown color and the cell nuclei were counterstained with hematoxylin. The positive signal was analyzed semiqualitatively automatically using the absorbance (A) value by a pathology image analysis system (PIPS-2020).

\section{Reverse Transcriptase-Polymerase Chain Reaction Analysis} for Klotho

Total RNA was extracted from frozen kidneys using TRIzol (Invitrogen, USA) according to the manufacturer's instructions. Firststrand cDNA was synthesized from RNA with oligo-d(T) primer and M-MLV reverse transcriptase (Promega, Madison, Wisc., USA). We amplified the synthesized first-strand cDNA by polymerase chain reaction (PCR) using the following primers: rat Klotho 5'-CAATGGCTTCCCTCCTTTAC-3' and 5'-AGCACAGGTTTGCGTAGTCT- $3^{\prime}$, rat $\beta$-actin 5'-ACCCACACTGTGCCCATCTATGA- ${ }^{\prime}$ and $5^{\prime}$-CATCGGAACCGCTCATTGCCGATAG$3 \prime$. The PCR products were electrophoresed on agarose gel (1.5\%) and visualized after ethidium bromide staining. DNA bands were analyzed by computer-assisted densitometric scanning. Band intensity was quantified by image analyzer (Ras-1000; Fuji, Japan).

\section{Western Blotting Analysis for Klotho and NADPH Oxidase}

Subunits Protein

Tissues were lysed in protein extraction buffer (Sigma, St. Louis, Mo., USA) and centrifuged at 13,000 rpm for $20 \mathrm{~min}$ after storing for $30 \mathrm{~min}$ on ice. Protein concentration was determined by the Bradford method (BioRad, Hercules, Calif., USA), protein (30-40 $\mu \mathrm{g}$ ) was applied to sodium dodecyl sulfate-polyacrylamide gel electrophoresis (SDS-PAGE) after degeneration for $5 \mathrm{~min}$ at $100^{\circ} \mathrm{C}$. Coomassie blue staining was used to ensure that equivalent amounts of protein were blotted, the protein was then transferred onto polyvinylidene fluoride (PVDF) membranes and blocked by PBST (PBS with $0.1 \%$ Tween-20) solution containing 5\% non-fat milk, and incubated with primary antibodies against Klotho (1:300; US Biology, USA), Nox-4 (1:200; SCB Inc.), NADPH-p47phox (1:200; SCB Inc.), NADPH-p22phox (1: 300; SCB Inc.) and $\beta$-actin (1:50,000; Sigma, USA), respectively at $4^{\circ} \mathrm{C}$ overnight. They were then incubated with their appropriate horseradish peroxidase-conjugated secondary antibodies (1:2,000; SCB Inc.) for $1 \mathrm{~h}$. Bands were developed using an ECL Western blotting detection system (Amersham). 
Table 1. Regular data before therapy (22 weeks, $\mathrm{n}=8$ )

\begin{tabular}{lcccc}
\hline & WKY group & SHR group & Fos group & Los group \\
\hline BP, mm Hg & $107.12 \pm 12.23$ & $192.03 \pm 9.79^{*}$ & $184.78 \pm 16.25^{*}$ & $187.26 \pm 13.86^{*}$ \\
Upro, mg/24h & $10.79 \pm 8.52$ & $78.94 \pm 27.15^{*}$ & $85.73 \pm 19.42^{*}$ & $82.03 \pm 34.13^{*}$ \\
NAGase, U/l & $12.03 \pm 7.45$ & $46.52 \pm 10.01^{*}$ & $39.56 \pm 11.32^{*}$ & $41.78 \pm 6.88^{*}$ \\
\hline
\end{tabular}

* Significantly different from the WKY group $(\mathrm{p}<0.05)$.

Table 2. Regular data after therapy ( 30 weeks, $n=8$ )

\begin{tabular}{lcccc}
\hline & WKY group & SHR group & Fos group & Los group \\
\hline BP, mm Hg & $110.47 \pm 17.87$ & $185.34 \pm 9.48^{*}$ & $135.30 \pm 20.33^{\#}$ & $128.89 \pm 12.86^{\#}$ \\
Upro, mg/24 h & $13.76 \pm 5.72$ & $101.01 \pm 23.42^{*}$ & $65.69 \pm 18.85^{\#}$ & $59.26 \pm 31.09^{\#}$ \\
NAGase, U/l & $14.89 \pm 8.57$ & $63.56 \pm 10.35^{*}$ & $47.25 \pm 12.03^{\#}$ & $50.74 \pm 7.12^{\#}$ \\
Scr, $\mu \mathrm{mol} / \mathrm{l}$ & $32.24 \pm 12.24$ & $55.71 \pm 8.79^{*}$ & $41.56 \pm 15.02^{\#}$ & $45.74 \pm 9.61^{\#}$ \\
BUN, $\mathrm{mmol} / \mathrm{l}$ & $5.98 \pm 1.12$ & $9.10 \pm 0.84^{*}$ & $8.32 \pm 1.65^{\#}$ & $8.18 \pm 0.97^{\#}$ \\
\hline
\end{tabular}

* Significantly different from the WKY group $(\mathrm{p}<0.05)$.

\# Significantly different from the SHR group $(\mathrm{p}<0.05)$.

Statistical Analyses

All results are presented as mean \pm SE and evaluated by multiple comparison tests and one-way analysis of variance (ANOVA). Correlation analyses were performed with Pearson's correlation. Statistical significance was set at $\mathrm{p}<0.05$.

\section{Results}

\section{Whole Animal Data}

Data are shown in tables 1 and 2. At 22 weeks, the blood pressure, NAGase, and Upro of SHR were obviously higher than in the WKY rats, and there was no significant difference between the three groups of SHR before therapy. At 30 weeks, the blood pressure, NAGase, Upro, Scr and BUN of the untreated SHR were significantly higher than in the WKY rats. Fos or Los reduced NAGase, Upro, Scr and BUN in SHR. Hypertension of SHR was controlled after treatment with Fos or Los.

\section{Renal Histology}

The morphological appearance of kidneys remained normal in the WKY group compared with untreated SHR. Kidneys in untreated SHR demonstrated arteriolar wall thickening and hyalinosis, interstitial edema with inflammatory cell infiltration, some dilated tubules with proteinaceous or cellular casts, and elevated collagenous components in the interstitium of the renal cortex, which showed typical pathological characteristics of hypertensive renal damage. Fos or Los treatment attenuated these lesions in SHR (fig. 1). The tubulointerstitial damage index in untreated SHR was significantly higher than in the WKY group. This index was obviously lower after treatment with Fos or Los.

\section{Immunohistochemical Detection of Klotho}

Immunostaining of the renal cortex showed that Klotho protein was localized in the cytoplasm and cellular nucleus of the renal tubules, predominantly in distal tubules: some proximal tubular cells were also positive (fig. 2). There was a significant degree of Klotho protein expression in the cortical tubules of WKY rats, while positive staining of Klotho protein expression was decreased in untreated SHR. Positive staining was elevated in SHR treated with Fos or Los compared with untreated SHR. There was no significant difference between these two drugs with regard to their effect on Klotho protein expression (fig. 2). 

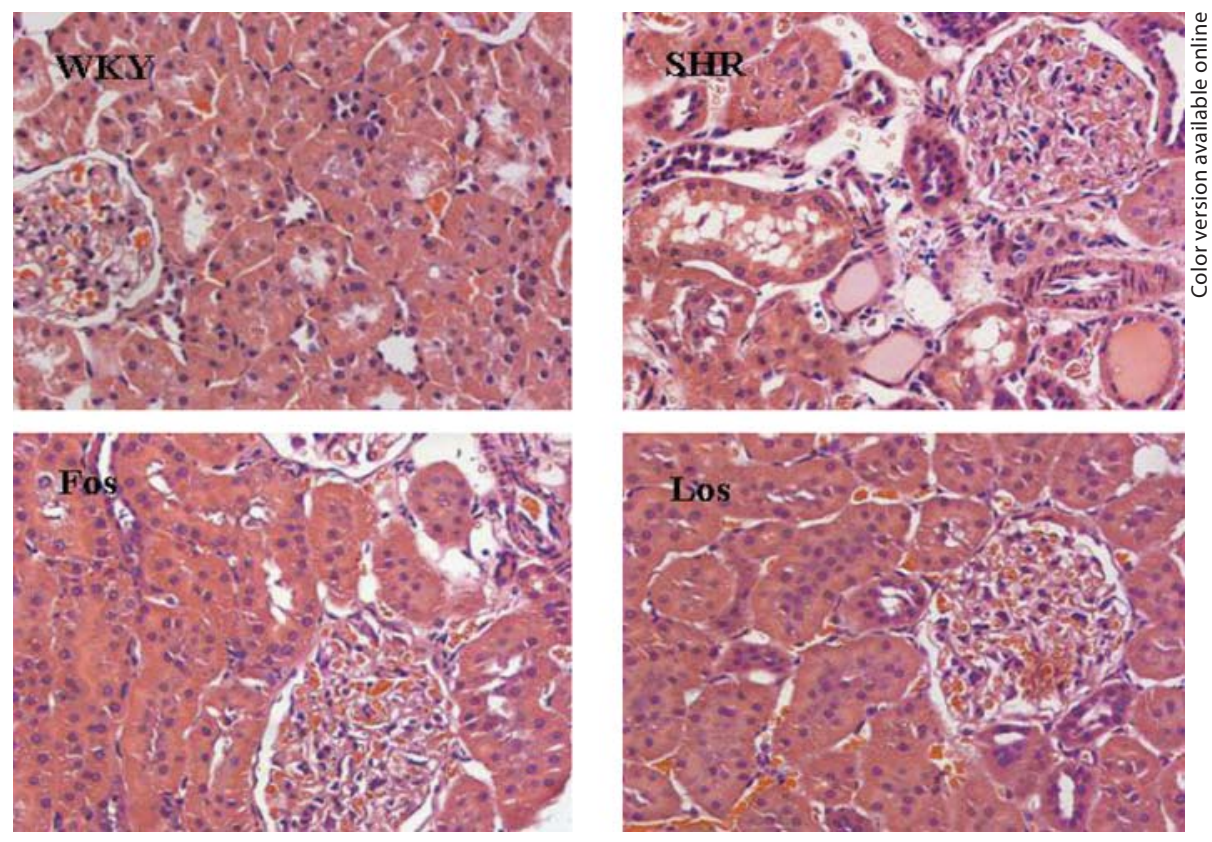

Fig. 1. HE staining of the kidneys of 30 -week-old WKY and SHR rats (original magnification $\times 400)$. Values are mean \pm SE $(n=8) .{ }^{*}$ Significantly different from the WKY group $(\mathrm{p}<0.05)$. " Significantly different from the SHR group $(\mathrm{p}<0.05)$.

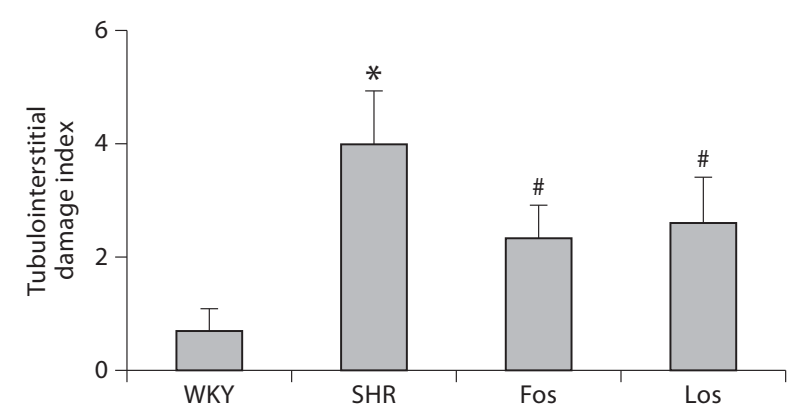

\section{Reverse Transcriptase-PCR Detection of Klotho}

Expression in the Renal Cortex

Compared with WKY rats, Klotho mRNA expression was significantly reduced in kidneys of untreated SHR. After treatment with Fos or Los, Klotho mRNA expression was evidently upregulated. However, there was no statistical difference between these two drugs regarding their effect on Klotho mRNA expression (fig. 3).

\section{Western Blotting Detection of Klotho and NADPH}

Oxidase Subunits Protein Expression in the Renal

Cortex

Renal Klotho protein expression was significantly inhibited in untreated SHR compared with WKY rats, concomitant with the increased protein levels of all three
NADPH oxidase subunits, Nox-4, p47phox and p22phox. The treatments with Fos or Los evidently elevated Klotho expression, while reducing the protein expression of all three NADPH oxidase subunits in kidneys of SHR. Furthermore, there was no statistical difference between the two drugs (fig. 4).

\section{Correlations}

Klotho protein expression was negatively related to the protein expression of NADPH oxidase subunit Nox-4 $(\mathrm{r}=-0.642 ; \mathrm{p}<0.05)$. Moreover, a negative correlation was also found between Klotho and NADPH-p47phox protein expression $(\mathrm{r}=-0.539 ; \mathrm{p}<0.05)$. Likewise, Klotho showed a significantly negative correlation with NADPHp22phox protein expression $(r=-0.703 ; p<0.05)$. 

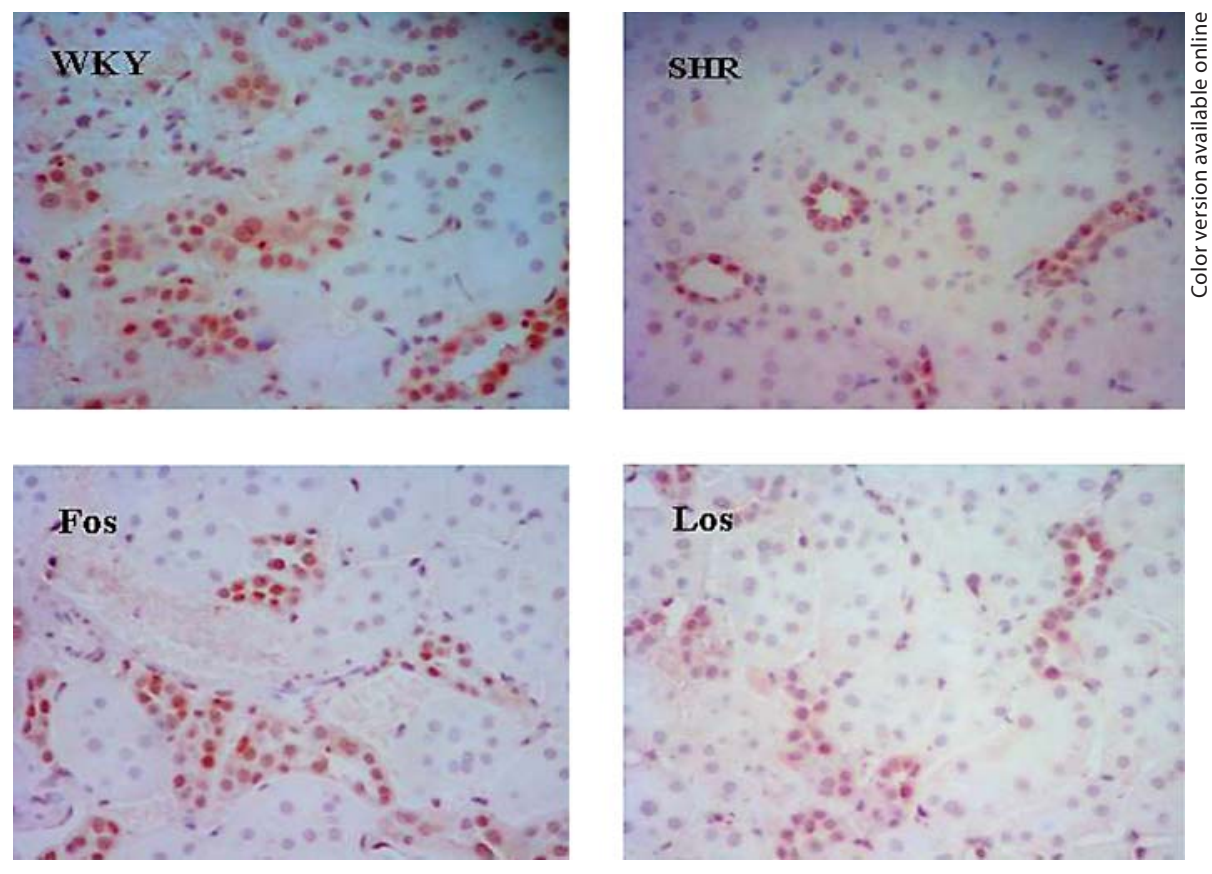

Fig. 2. Immunohistochemical detection of Klotho protein expression in the renal cortex (original magnification $\times 400$ ). Values are mean $\pm \mathrm{SE}(\mathrm{n}=8)$. * Significantly different from the WKY group $(p<0.05)$. \# Significantly different from the SHR group $(\mathrm{p}<0.05)$.

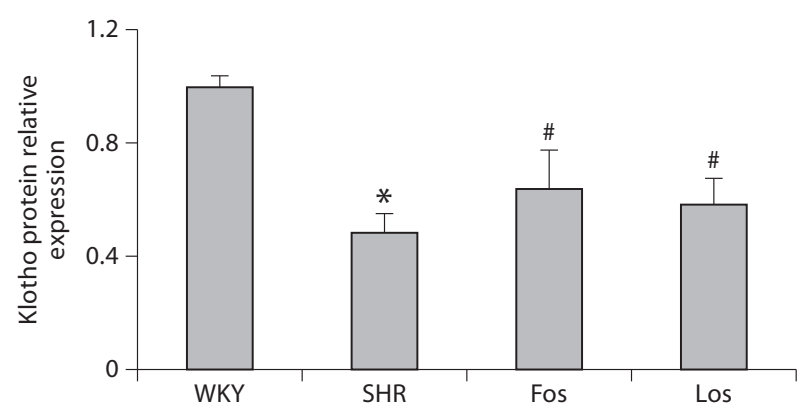

Fig. 3. mRNA expression of Klotho in the renal cortex detected by reverse transcriptase-PCR analysis. Values are mean \pm SE $(\mathrm{n}=8) .{ }^{*}$ Significantly different from the WKY group $(\mathrm{p}<0.05)$. " Significantly different from the SHR group $(\mathrm{p}<0.05)$.
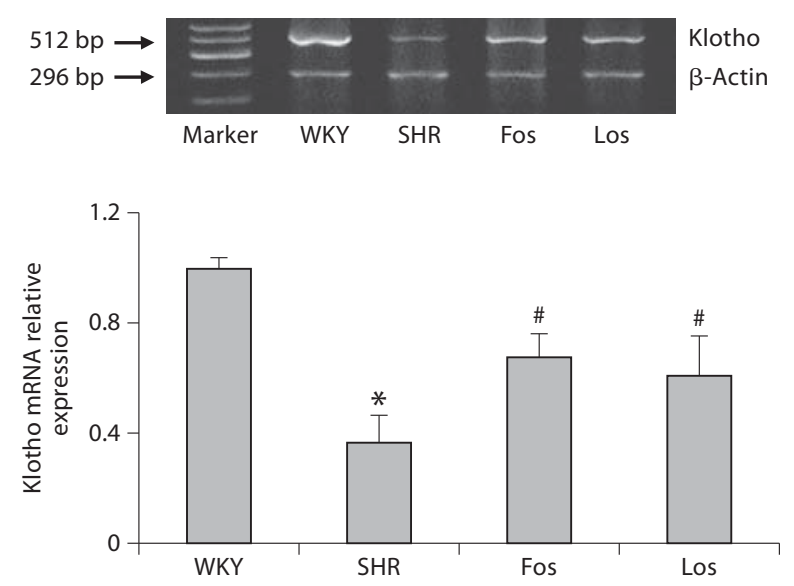


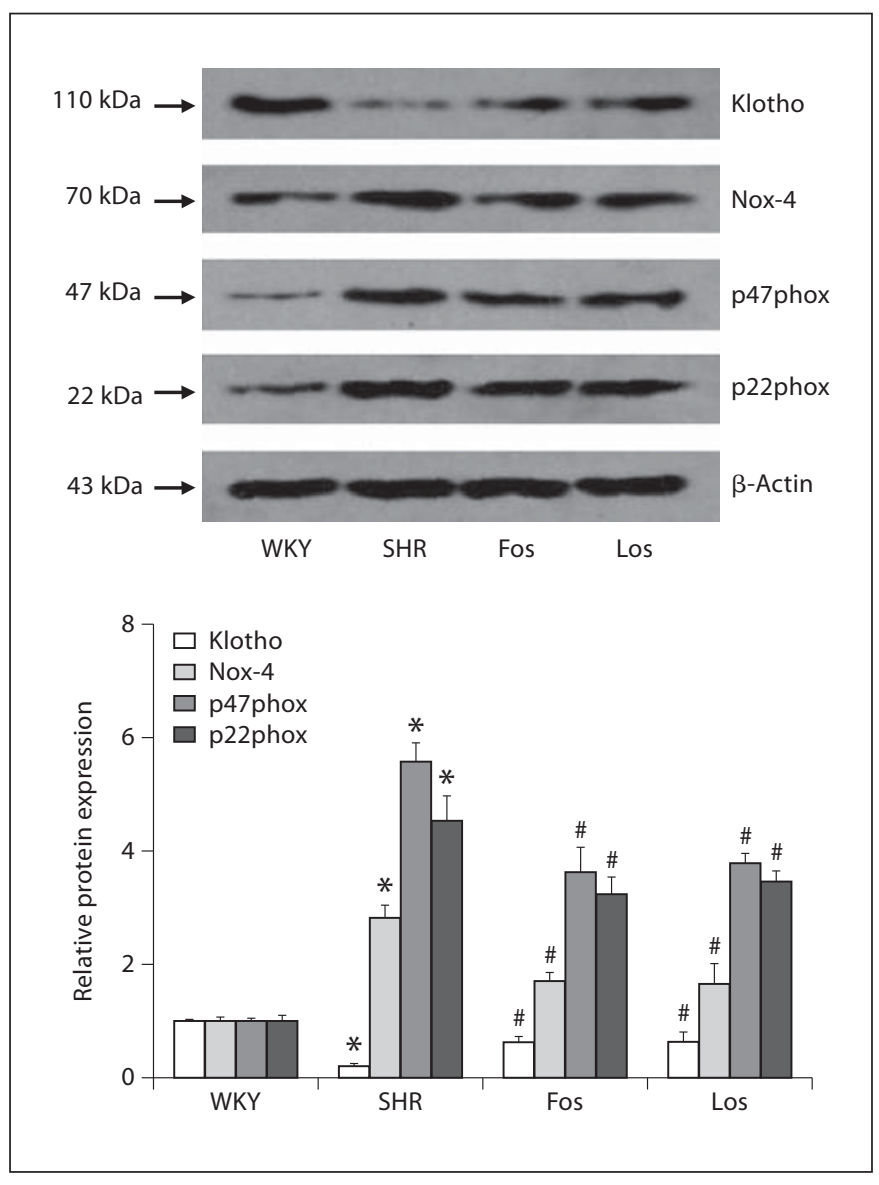

Fig. 4. Western blotting detection of Klotho and NADPH oxidase subunit protein expression in the renal cortex. Values are mean \pm SE $(n=8)$. * Significantly different from the WKY group $(\mathrm{p}<$ $0.05)$. ${ }^{\#}$ Significantly different from the SHR group $(\mathrm{p}<0.05)$.

\section{Discussion}

In the present study, we demonstrated three major findings. Firstly, Klotho mRNA and protein expression were reduced, and NADPH oxidase protein expression were elevated in kidneys of SHR. Secondly, Fos or Los increased Klotho expression reduced in kidneys of SHR, and decreased NADPH oxidase expression, which might be a novel renoprotective mechanism involving Fos and Los in hypertensive renal damage. Finally, correlation analyses showed that there were negative correlations between Klotho and NADPH oxidase subunits expression, indicating that the NADPH oxidase might be downstream of the Klotho gene.

We found that Klotho mRNA and protein expression were detected in established cultured cell lines NRK-52E in our previous findings [16]. To confirm the findings in vitro, we further investigated Klotho expression in kidneys of SHR, the age-matched WKY serving as controls. SHR is a widely used animal model, especially for human essential hypertension. We found that Klotho gene expression was reduced significantly in kidneys of SHR, which was consistent with the previous study [6]. Moreover, SHR can progress to end-organ damage including renal failure and heart failure. In hypertensive nephropathy, the typical initial renal pathological changes of SHR are detectable and obvious in the inner cortex, and RAS plays a crucial role in this model [17]. The present study showed that the systolic blood pressure of 22-week-old SHR was obviously higher than that of WKY rats, and continued to increase with age. Compared to the WKY group, there was obvious hypertensive renal pathologic damage, and significant elevation of NAGase, Upro, Scr and BUN in 30-week-old SHR.

Studies reported that Klotho gene overexpression increased resistance to oxidative stress in several types of cells $[18,19]$. The antioxidative mechanisms of Klotho gene may be through suppression of the insulin/IGF-1 pathway, activation of cAMP, or increment of NO [20]. Because of the important relationship between Klotho gene and oxidative stress, we have focused on renal oxidative stress in SHR. Renal oxidative stress has been detected in several hypertension models $[21,22]$. Experimental studies have established a role for oxidative stress in hypertensive renal damage and the therapeutic potential of antioxidant treatment $[23,24]$. NADPH oxidase, the major source of ROS, mainly consists of membrane component (p22phox, gp91phox, Rap1A) and cytoplasmic component (p47phox, p67phox, p40, Rac2) [25]. Our data revealed that renal protein expression of NADPH oxidase subunits, Nox-4, p47phox and p22phox in SHR was significantly increased compared with that of WKY rats, indicating the elevation of NADPH oxidase in kidneys of SHR. This observation has confirmed the occurrence of renal oxidative stress in SHR.

The importance of RAS in the pathogenesis and progression of hypertensive renal damage was recognized long ago. Blockade of the RAS with ACEI and/or ARB is currently the best documented treatment strategy to attenuate the progression of hypertensive renal damage [26-28]. Therefore, we adopted Fos or Los for intervention in SHR, finding that Fos or Los could decrease blood pressure, NAGase, proteinuria, Scr and BUN, and renal pathological damage. These data indicated that Fos and Los were renoprotective drugs in hypertensive renal damage. Proteinuria is one of the most important and 
independent risk factors for the progression of renal diseases [29]. The reduction of proteinuria was partly related to blood pressure control. Our work demonstrated that Fos or Los alleviated the development of spontaneous hypertension and renal damage in SHR.

To determine the mechanisms of renoprotective effects of Fos and Los, we further studied the effects of Fos and Los on renal Klotho and NADPH oxidase expression in vivo. There have been no previous studies on the influence of Fos and Los on renal Klotho expression in vivo. Currently, studies reported that continuous infusion of angiotensin II (Ang II) in rats downregulated renal Klotho expression mediated by AT1 receptor; exogenous delivered Klotho gene could ameliorate tubulointerstitial damage induced by Ang II administration [30]. The results of the present study provide new insights into renoprotective effects of Fos and Los. We found that Fos or Los attenuated the reduction of Klotho gene expression, whilst reducing the upregulation of all three NADPH oxidase subunits, Nox-4, p47phox and p22phox expression in kidneys of SHR.

In addition, our correlation study demonstrated that Klotho protein expression was negatively correlated with all three NADPH oxidase subunits protein expressions. Wang and Sun [6] found that Klotho gene delivery could abolish the increment of NADPH oxidase activity, and the renoprotective effect of Klotho might be attributed in part to inhibiting Nox-2 and superoxide production in SHR. In our study, the reduction of NADPH oxidase expression in the Fos or Los group might be due in part to the increment of Klotho, which suggested NADPH oxidase action occurred downstream of the Klotho gene, although the precise mechanism remains to be found. The results suggested that Fos or Los could elevate renal Klotho gene expression, thus attenuating renal oxidative stress and slowing the progression of hypertensive renal damage.

The limitations of our study should be acknowledged. Although we found that Fos or Los elevated renal Klotho expression and inhibited NADPH oxidase expression, the causal relationship between Klotho and NADPH oxidase expression was not proved in this study. In vivo Klotho knock down/out or gene transfer should be performed in the following study to prove this relationship.

In summary, our results show that Fos and Los provide protection from hypertensive renal damage, the effects probably being mediated by enhancement of antiaging gene Klotho expression and inhibition of NADPH oxidase expression.

\section{References}

1 Kuro-o M, Matsumura Y, Aizawa H, et al: Mutation of the mouse Klotho gene leads to a syndrome resembling ageing. Nature 1997; 390:45-51.

2 Masuda H, Chikuda H, Suga T, et al: Regulation of multiple ageing-like phenotypes by inducible Klotho gene expression in Klotho mutant mice. Mech Ageing Dev 2005;126: 1274-1283.

3 Li SA, Watanabe M, Yamada H, et al: Immunohistochemical localization of Klotho protein in brain, kidney, and reproductive organs of mice. Cell Struct Funct 2004;29:9199.

-4 Sugiura H, Yoshida T, Mitobe M, et al: Klotho reduces apoptosis in experimental ischaemic acute kidney injury via HSP-70. Nephrol Dial Transplant 2010;25:60-68.

$\checkmark 5$ Koh N, Fujimori T, Nishiguchi S, et al: Severely reduced production of Klotho in human chronic renal failure kidney. Biochem Biophys Res Commun 2001;280:1015-1020.

-6 Wang Y, Sun Z: Klotho gene delivery prevents the progression of spontaneous hypertension and renal damage. Hypertension 2009;54:810-817.
7 Cheng MF, Chen LJ, Cheng JT: Decrease of Klotho in the kidney of streptozotocin-induced diabetic rats. J Biomed Biotechnol 2010;2010:513853.

8 Haruna Y, Kashihara N, Satoh M, et al: Amelioration of progressive renal injury by genetic manipulation of Klotho gene. Proc Natl Acad Sci USA 2007;104:2331-2336.

$\checkmark 9$ Yahata K, Mori K, Arai H, et al: Molecular cloning and expression of a novel Klotho-related protein. J Mol Med 2000;78:389-394.

10 Kuro-o M: Klotho as a regulator of oxidative stress and senescence. Biol Chem 2008;389: 233-241.

11 Yamamoto M, Clark JD, Pastor JV, et al: Regulation of oxidative stress by the anti-aging hormone Klotho. J Biol Chem 2005;280: 38029-38034.

12 Tyther R, Ahmeda A, Johns E, et al: Proteomic profiling of perturbed protein sulfenation in renal medulla of the spontaneously hypertensive rat. J Proteome Res 2010;9: 2678-2687.

13 Sedeek M, Hébert RL, Kennedy CR, et al: Molecular mechanisms of hypertension: role of Nox family NADPH oxidases. Curr Opin Nephrol Hypertens 2009;18:122-127.
14 Macconi D: Targeting the renin-angiotensin system for remission/regression of chronic kidney disease. Histol Histopathol 2010;25: 655-668.

15 Kobori H, Nangaku M, Navar LG, et al: The intrarenal renin-angiotensin system: from physiology to the pathobiology of hypertension and kidney disease. Pharmacol Rev 2007;59:251-287.

16 Zhou Q, Lin S, Tang R, Veeraragoo P, et al: Role of fosinopril and valsartan on Klotho gene expression induced by angiotensin II in rat renal tubular epithelial cells. Kidney Blood Press Res 2010;33:186-192.

17 Kobori H, Ozawa Y, Suzaki Y, et al: A. Enhanced intrarenal angiotensinogen contributes to early renal injury in spontaneously hypertensive rats. J Am Soc Nephrol 2005;16: 2073-2080.

18 Rakugi H, Matsukawa N, Ishikawa K, et al: Anti-oxidative effect of Klotho on endothelial cells through cAMP activation. Endocrine 2007;31:82-87.

19 Mitobe M, Yoshida T, Sugiura H, et al: Oxidative stress decreases Klotho expression in a mouse kidney cell line. Nephron Exp Nephrol 2005;101:e67-e74 
20 Kuro-o M: Klotho. Pflugers Arch 2010;459: 333-343.

-21 Lopez-Ruiz AF, Iliescu R, Reckelhoff JF: Refractory blood pressure in female SHR to increased oxidative stress is not mediated by $\mathrm{NO}$ or by upregulation of renal antioxidant enzymes. Am J Physiol Regul Integr Comp Physiol 2010;298:266-271.

22 Wang Y, Chen AF, Wang DH: Enhanced oxidative stress in kidneys of salt-sensitive hypertension: role of sensory nerves. Am J Physiol Heart Circ Physiol 2006;291:31363143.

23 Yen PL, Chen BH, Yang FL, et al: Effects of deep-frying oil on blood pressure and oxidative stress in spontaneously hypertensive and normotensive rats. Nutrition 2010;26: 331-336.
24 Hou X, Shen YH, Li C, et al: PPAR $\alpha$ agonist fenofibrate protects the kidney from hypertensive injury in spontaneously hypertensive rats via inhibition of oxidative stress and MAPK activity. Biochem Biophys Res Commun 2010;394:653-659.

25 Gill PS, Wilcox CS: NADPH oxidases in the kidney. Antioxid Redox Signal 2006;8:15971607.

26 Onuigbo MA: Analytical review of the evidence for renoprotection by renin-angiotensin-aldosterone system blockade in chronic kidney disease - a call for caution. Nephron Clin Pract 2009;113:c63-c69.
27 Bakris G: Are there effects of renin-angiotensin system antagonists beyond blood pressure control? Am J Cardiol 2010;105:21-29.

28 Liu XP, Pang YJ, Zhu WW, et al: Benazepril, an angiotensin-converting enzyme inhibitor, alleviates renal injury in spontaneously hypertensive rats by inhibiting advanced glycation end-product-mediated pathways. Clin Exp Pharmacol Physiol 2009;36:287296.

29 Jafar TH, Stark PC, Schmid CH, et al: Proteinuria as a modifiable risk factor for the progression of non-diabetic renal disease. Kidney Int 2001;60:1131-1140.

-30 Mitani H, Ishizaka N, Aizawa T, et al: In vivo Klotho gene transfer ameliorates angiotensin II-induced renal damage. Hypertension 2002;39:838-843. 